Ann. Abeille, 1965, 8 (2), Iog-III.

\title{
OBSERVATIONS SUR UN SITE DE NIDIFICATION DE MELITTURGA CLAVICORNIS LATR. HYMENOPT. APOIDEA
}

\author{
S. TIRGARI \\ Station de Recherches sur l'Abeille et les Insectes sociaux \\ Bures-sur-Yvette, (Seine-et-Oise)
}

SOMMAIRE

L'auteur expose le résultat d'observations effectuées sur un site de nidification de Melitturga clavicornis LATR. situé en Suisse.

\section{INTRODUCTION}

La protection des espèces pollinisatrices et les essais d'acclimatation dans les zones où elles n'existent pas, se heurtent aux lacunes considérables qui existent encore en ce qui concerne les points très élémentaires de leur biologie. Ainsi, notre connaissance des conditions de nidification est presque nulle pour de nombreuses espèces et, d'autre part, l'étude de la phase du cycle biologique de l'insecte qui constitue l'hivernage reste à faire dans la plupart des cas.

Dans ces conditions, nous avons profité avec empressement de l'occasion qui nous a été offerte en septembre 1964 d'étudier un site de nidification d'un insecte connu comme excellent pollinisateur de la Luzerne : Melitturga clavicornis LATr.

\section{SITUATION GÉOGRAPHIQUE DU STTE}

Les nids se trouvent situés dans un terrain de camping d'environ mille cinq cents mètres carrés se trouvant aux environs de Sierre dans le Valais (Suisse).

Ce terrain est situé à peu de distance des rives du Rhône, sur le versant sud de la vallée.

L'exposition du terrain est ensoleillée, en été, de 9 heures à une heure très avancée. 
Le sol est recouvert d'une régétation très clairsemée ; par endroit, il est même complètement nu.

A l'époque de ma visite, deuxieme semaine de septembre, aucun nid n'était en activité, mais, selon plusieurs témoignages, les Melitturga y avaient été très actifs tout l'été et nidifient dans ce lieu, au moins depuis 1924 .

\section{CARACTÉRISTIQUES DU SOI,}

La surface du sol était particulièrement dure ct sc̀che, l'été ayant été très peu pluvieux. Une couche d'argile de 5 à Io centimètres d'épaisseur occupait la surface et recouvrait un sol sableux. mélangé de pierres d'assez grosse taille sans aucun gravier. Le sol, par son aspect, rappelait considérablement les sols adoptés par l'abeille alkali (Nomia melanderi) dans le sud de l'śtat d'Utah, U. S. A.

Une analyse effectuée au laboratoire d'analyse des sols de l'I. N. R. A. à Pont-de-la-Maye a donné les résultats suivants :

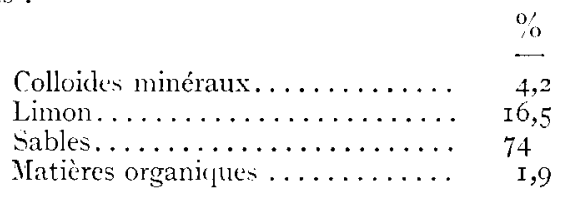

RECHERCHES DES NIDS E'T RÉSULTATS

Durant les trois jours qu'il nous a été possible de consacrer à ce travail, nous avons creusé trente-deux trous d'un mètre carré de surface et cinquante centimètres de profondeur, répartis sur l'ensemble du terrain de camping.

Cette fouille nous a permis de recueillir 22 larves de Melitturga clavicomis. Les larves se trouvaient dans des cellules horizontales situées entre 5 et 35 centimètres de la surface du sol, cependant la majorité se trouvait entre 20 et $30 \mathrm{~cm}$.

A cette occasion, nous avons également découvert le nid d'une autre espèce pollinisatrice: Melitta leporina $\mathrm{Pz}$ dans une portion du site couverte de végétation. Dans ce nid, se trouvait dix cocons, placés verticalement en deux rangées, très près les uns des autres et à vingt centimètres de la surface.

Nous avons également mis à jour un très grand nombre de nids d'un Sphecida, chasseur d'abeille: Philanthus triangulum F.

Nous avons pu vérifier que ces nids ne contenaient que des débris d'Abeille domestique et que cette espèce ne s'attaquait pas aux Melitturga.

\section{CONCLUSIONS}

Ces quelques observations ont apporté quelques précisions sur les caractères de la nidification d'un insecte particulièrement intéressant par ses aptitudes à polliniser la Luzerne.

Malheureusement, ce site ne pourra plus être étudié, car il sera détruit avant la saison prochaine par les travaux de construction d'une autoroute. Nous citons ce détail, car il illustre bien le processus en cours d'élimination de la faune polli- 
nisatrice qui résiste nual aux modifications de plus en plus profondes apportées par l'homme au miliet.

Dans ces conditions, des mesures destinées à favoriser les pollinisateurs, par exemple la création de sites offrant les conditions les meilleures pour leur nidification, semblent s'imposer.

Reçu pour publication en auril 1965

\section{REMERCIEMENTS}

L'auteur exprime sa gratitude à M. le Professeur de Beaunont du Musée zoologique de Lausanne, qui a bien voulu le guider jusqu'au lieu de ce travail.

L'auteur remercie également le $\mathrm{D}^{\mathrm{r}}$ W. P. STEPIILN, Oregon state University, Corvalis, Oregon, U. S. A., qui a pris à sa charge les frais du déplacement nécessité par cette étude.

\section{SUMMARY}

COMMENTS ON A NIDIFICATION SITE OF “MELITTURGA CLAVICORNIS" LATR., HYMFNOPT. APOIDEA

The author had the opportunity of studying a nidification site of Meliturga claciomis LATR. which has been inhabited for more than forty years in the Rhone valley in Swizerland. 